\title{
Recent Advances in Spiro[fluorene-9,9'-xanthene]-Based Hole Transport Materials for Perovskite Solar Cells
}

\author{
Mingli Sun, ${ }^{a}$ Changjin Ou, ${ }^{b}$ Baoyi Ren, ${ }^{*, c}$ Linghai Xie, ${ }^{b}$ Xianghua Zhao, ${ }^{*, d}$ and Wei Huang ${ }^{b, e}$ \\ ${ }^{a}$ College of Chemistry, Chemical Engineering and Resource Utilization, Northeast Forestry University, \\ 26 Hexing Road, Harbin, Heilongjiang 150040, China \\ ${ }^{b}$ Key Laboratory for Organic Electronics and Information Displays \& Jiangsu Key Laboratory for Biosensors, \\ Institute of Advanced Materials (IAM), Jiangsu National Synergistic Innovation Center for Advanced Materials \\ (SICAM), Nanjing University of Posts \& Telecommunications, 9 Wenyuan Road, Nanjing 210023, China \\ ${ }^{c}$ College of Applied Chemistry, Shenyang University of Chemical Technology, Shenyang, \\ Liaoning 110142, China \\ ${ }^{d}$ College of Chemistry and Chemical Engineering, Xinyang Normal university, Xinyang, Henan 464000, China \\ ${ }^{\text {e }}$ Shaanxi Institute of Flexible Electronics (SIFE), Northwestern Polytechnical University (NPU), \\ 127 West Youyi Road, Xi'an, Shaanxi 710072, China
}

Email: baoyir@126.com (B. R.),4773zxh@163.com (X. Z.)

\begin{abstract}
The recent advances in perovskite solar cells (PSCs) surprised the traditional photovoltaic community. The power conversion efficiencies (PCEs) of PSCs have achieved more than $20 \%$, in which the key functional layer is currently constituted by spirobifluorene (SBF)-based hole transporting materials (HTMs), typically known as spiro-OMeTAD. In the meanwhile, a new rising spiro-core, spiro[fluorene-9,9'-xanthene] (SFX), has attracted great interest for contrasting HTMs. SFX exhibits two main advantages compared with SBF: one is low-cost, the other is high stability. Hence, a lot of highly efficient HTMs based on SFX have been designed and synthesized, and the corresponding PSCs devices have shown competitive performances with spiro-OMeTADbased cells. In this minireview, we summarize the structure-performance relationship of high-efficiency SFX-based HTMs for PSCs. The effects of the highest occupied molecular orbital (HOMO) energy level, hole mobility and conductivity, film-forming ability of SFX-based HTMs on the PCEs have been focused.
\end{abstract}

Keywords spiro[fluorene-9,9'-xanthene], perovskite solar cells, hole transport materials, power conversion efficiency, spiro-OMeTAD

\section{Introduction}

Solar energy, as a renewable and sustainable resource unlike fossil fuels, is being widely developed and applied in lots of modern technologies. Among these technologies, solar cell is the most promising one owing to pollution-free and inexhaustibility character. ${ }^{[1]}$ Currently, silicon-based solar cells still occupy the photovoltaic market due to their well-developed technology and high efficiency, outclassing other photovoltaic technologies such as organic-inorganic hybrid perovskite solar cells (PSCs), dye-sensitized solar cells (DSSCs) and organic photovoltaics (OPV). Among them, PSCs have created much excitement in the solar cell community. ${ }^{[2-5]}$ The power conversion efficiency (PCE) of PSCs has increased from $4 \%$ to $22 \%$ in the last 7 years. ${ }^{[6]}$ Due to their promising power efficiencies and outstanding features, PSCs are expected to surpass traditional crystalline silicon-based solar cells.

Generally, the structure of PSCs includes two types planar structure (Figure 1a) and mesoscopic structure (Figure 1b). The former has the advantage of a simple architecture with a thin layer of perovskite sandwiched between the hole and electron transport layers. The latter is thought to enhance charge collection by decreasing the carrier transport distance, preventing direct current leakage and increasing photon absorption due to light scattering. ${ }^{[7]}$ In these devices, $\mathrm{TiO}_{2}$ or other metallic oxide is used to collect and transport the electrons, perovskite compounds are used as a light-harvesting active layer, hole transport materials (HTMs) are used as hole conductors, which

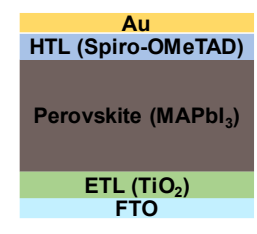

(a) planar structure

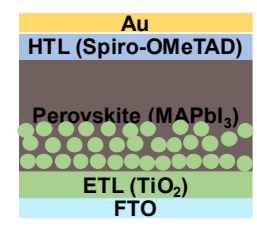

(b) mesoscopic structure

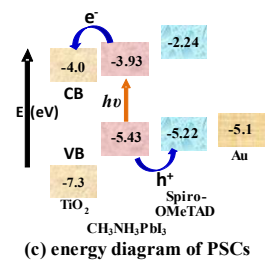

(c) energy diagram of PSCs
Figure 1 Schematic diagram of perovskite solar cells (a) planar structure (b) mesoscopic structure (c) energy diagram of PSCs.

act as the electron-blocking layer minimizing charge recombination and improve the direct contacts between the perovskites and metal electrodes as well as extract and transport holes (Figure 1c). The properties of HTMs such as energy level, conductivity and charge mobility play an important role in PSCs for the high and stable device performance. In order to further improve the photovoltaic performance and stability of PSCs, it is important to develop high-performance HTMs. Many small molecules, polymers and inorganic salts have been explored as HTMs in PSCs, but small molecular materials are more popular due to their defined molecular structure and easy purification. For example, carbazole derivatives, ${ }^{[8-10]}$ triphenylamine derivatives ${ }^{[11,12]} 4,4$ '-dimethoxy- diphenylamine derivatives, ${ }^{[1,12]}$ butadiene derivatives, ${ }^{[13]}$ and various thiophene derivatives, ${ }^{[14-19]}$ have been designed and utilized owing to their excellent charge 
carrier transport property. Among these materials, 2,2',7,7'tetrakis-( $N, N$-di-p-methoxyphenyl-amine)-9,9'-spirobifluorene (spiro-OMeTAD, Figure 2) is so far the most recognized and commonly used HTM in PSCs due to its amorphous nature, high solubility in organic solvents, ${ }^{[20]}$ good pore filling of the $\mathrm{TiO}_{2}$ film, low recombination rates and efficient charge transport. To date, most high efficiencies (> 18\% PCE) of PSCs have been obtained by using spiro-OMeTAD as HTM. ${ }^{[21-23]}$ However, the complex synthetic route and purification of the 2,2',7,7'-tetrabromo-9,9'-spirobi- [fluorene] (4Br-SBF) core lead to a high cost to obtain spiro-OMeTAD, which limits the large-scale application of spiro-OMeTAD in the coming commercialized PSCs. For the sake of lower-cost materials, many new organic HTMs have been designed and applied in PSCs to try to replace spiroOMeTAD. Because of the similar structure and property to 9,9'-spirobi-[fluorene] (SBF), spiro[fluorene-9,9'-xanthene] (SFX) has also been used as a core to design novel HTMs for PSCs. Economic and concise one-pot synthesis of brominated SFX derivatives $^{[24]}$ makes SFX-based HTMs very promising for large-scale industrial production in the future. In this article, recent progress of SFX-based HTMs applied in PSCs were reviewed. Their structural and opto-electronic properties as well as effects on the performance of PSCs devices are addressed.

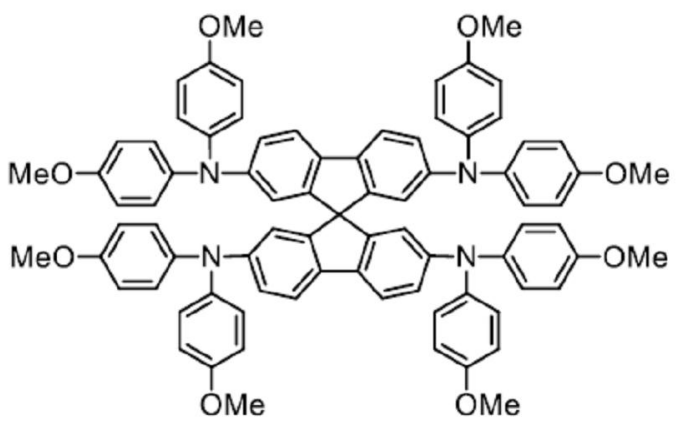

Figure 2 The structure of spiro-OMeTAD.

\section{Low-Cost Synthesis of SFX-Based Derivatives}

HTMs used to construct PSCs should have the advantages of low-cost preparation and good solubility for solution-processed devices. The spiro-compounds with perpendicular architecture can effectively inhibit the intermolecular $\pi-\pi$ stacking and thus have good solubility, which is beneficial to the fabrication of the devices using the wet chemical process such as spin-coating. Therefore, lots of them are applied in the opto-electronic field of large area processing. For example, SBF-cored derivatives such as spiro-OMeTAD are often used as HTMs in PSCs.

Currently, most of SBF-based HTMs are synthesized from brominated SBF intermediates, whose cost directly determines the cost of SBF-based HTMs. Generally, the synthetic method of SBF originates from Clarkson's synthesis (Figure 3 ) in the earliest stage, which needs three steps. ${ }^{[25]}$ Subsequently, brominated SBF intermediates are obtained by some conventional bromination reaction (Figure 4). However, the bromination site of SBF is simple, only at the 2,7 positions, which leads to a reduced number of SBF-based isomers. It is not easy to control and separate for obtaining mono brominated SBF or double

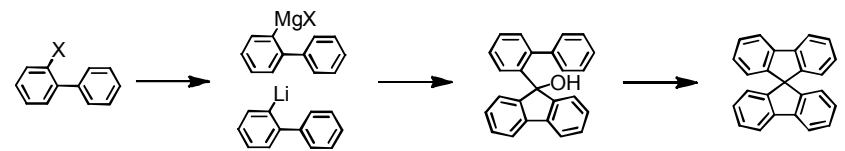

Figure 3 The synthetic route to 9,9'-spirobifluorene.

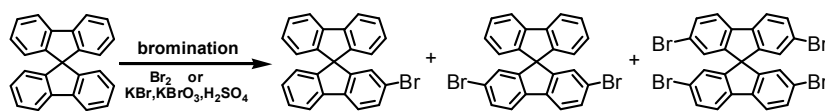

Figure 4 The synthetic method of brominated spirobifluorene intermediates.

brominated SBF singly. This undoubtedly increases the preparation cost of SBF-based semiconductor materials.

In contrast to SBF, SFX has received increasing attention as next-generation spiro-fluorene in the last ten years due to its competitive synthetic route ${ }^{[24]}$ and provided a design platform for organic semiconductor molecules design via various $\mathrm{C}-\mathrm{X}$ cross-coupling reactions and $\mathrm{C}-\mathrm{H}$ bond activation reactions. ${ }^{[26]}$ SFX, consisting of fluorene and xanthene moieties, can be synthesized by one-step reaction (Figure 5 ) and easily purified by ethanol without column chromatographic separation. The two different moieties connected by a $\mathrm{sp}^{3}$-hybridized carbon make SFX have more active sites, which also leads to more different brominated isomers. Various brominated SFX intermediates can be obtained with high yields by using different reaction materials of bromo-fluorenone and bromophenol (Figure 5). ${ }^{[26]}$ Unlike the symmetric SBF, the molecular designs of HTMs based on SFX are more abundant, selective and expectant. Meanwhile, the non-planar structure of SFX presents better 3D intermolecular interaction and electrical conductivity when SFX is introduced into HTM molecules. ${ }^{[27]}$

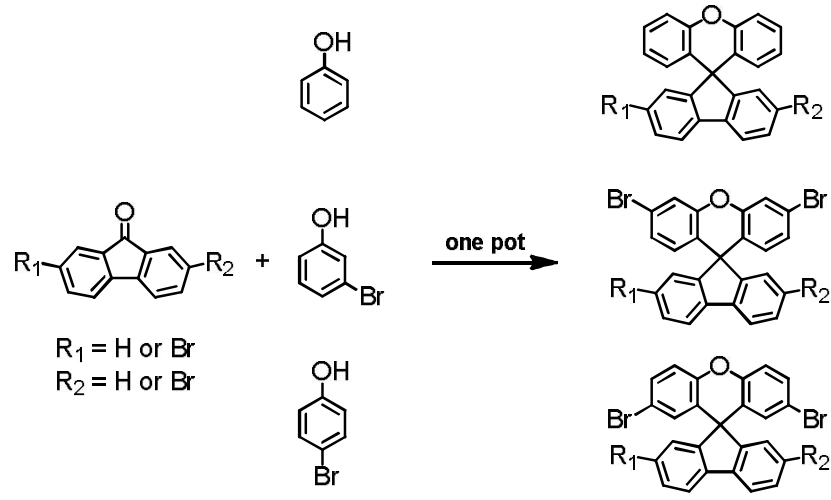

Figure 5 The synthetic route to brominated SFX intermediates.

\section{SFX-Based HTMs Applied in PSCs}

As HTMs in PSCs, suitable energy level matching with the perovskite, high conductivity and charge mobility, smooth and stable films are considered as important factors when designing molecules. ${ }^{[1]}$ The introduction of SFX with a nonplanar 3D architecture into hole-transporting molecules has a significant effect on the conductivity, energy levels, and thin-film surface morphology, which further affects the performance of PSCs.

Spiro-OMeTAD has been used as a hole transport material for solid-state dye solar cells (ssDSCs) and PSCs for a long time, but no one has ever developed a novel HTM based on another potential spiro-fluorene structure SFX. Until 2016, Sun and co-workers ${ }^{[28]}$ synthesized a low-cost SFX-based HTM termed X60 (Figure 6) with a structure extremely like spiroOMeTAD through two-step synthesis. Devices based on X60 as HTM showed high power conversion efficiency (PCEs) of up to $7.30 \%$ in ssDSCs and $19.84 \%$ in PSCs, which can be comparable with the reported record PCEs of $7.7 \%^{[29]}$ (ssDSCs) and $20.8 \%^{[21]}$ (PSCs) obtained by using spiro-OMeTAD as HTM. The properties of $X 60$ are not superior but only similar to spiroOMeTAD from molecular energy levels to hole mobility and conductivity as well as film-forming state. Since then, more 


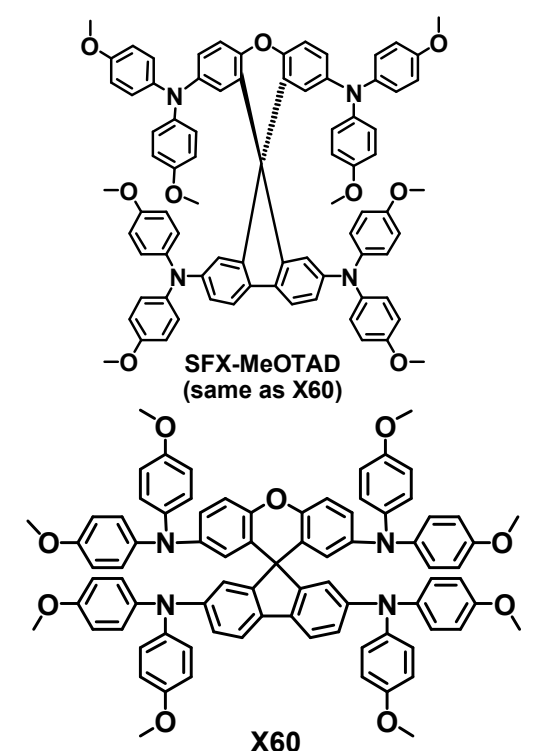

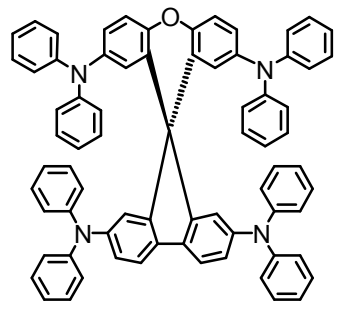

SFX-TAD

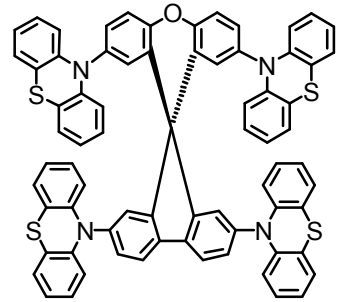

SFX-TPTZ

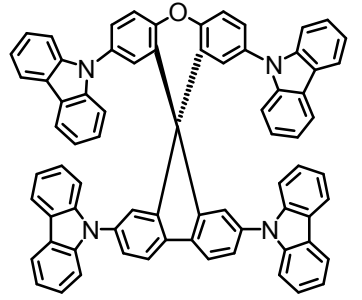

SFX-TCZ

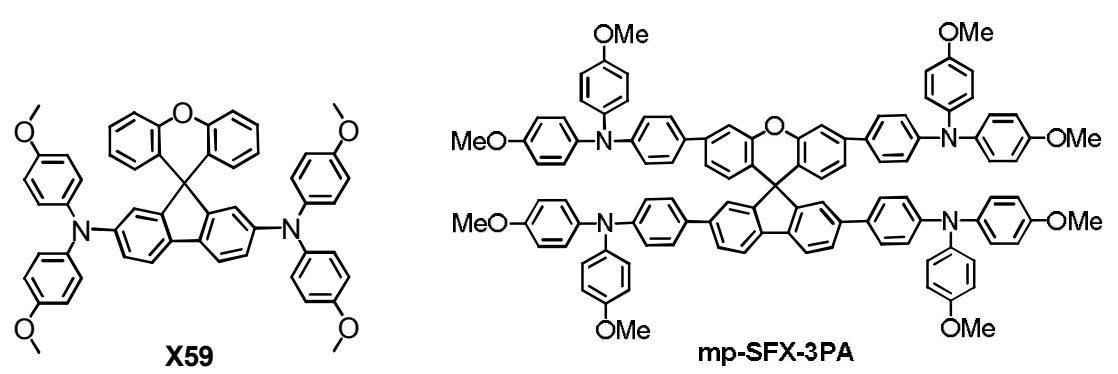

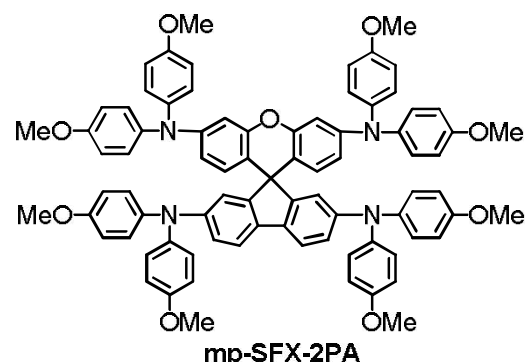

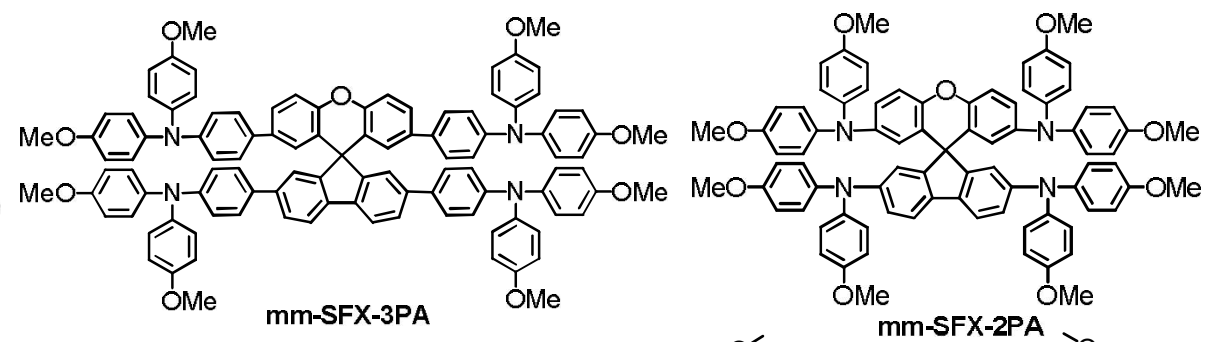

mm-SFX-3PA

$\mathrm{OMe}$

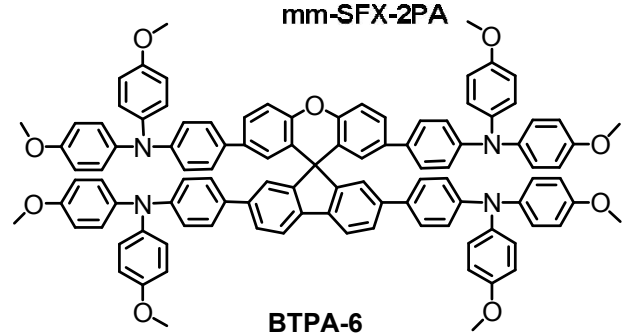

Figure 6 The structures of HTMs (1).

SFX-based HTMs applied in PSCs have been designed mainly based on the above three characteristics.

\section{Highest occupied molecular orbital (HOMO) energy level}

Maciejczyk et al. ${ }^{[30]}$ synthesized four novel triarylaminebased HTMs with SFX as core used in PSCs. Among them, SFX-MeOTAD (the same as X60) has the same HOMO level (about $-5.1 \mathrm{eV}$ ) as spiro-OMeTAD, which is higher than that of SFX-TCZ, SFX-TPTZ and SFX-TAD (Figure 6, -5.34, -5.70 and $-5.39 \mathrm{eV}$, respectively). Although the lower HOMO energy level could potentially lead to higher open circuit voltages of the operating devices, the authors only investigated SFX-MeOTAD in detail for application in PSCs and compared it with spiroOMeTAD due to the poor solubility of the latter three compounds. The results showed that the device based on SFX-MeOTAD exhibited high PCE up to $12.4 \%$, which was slightly lower than that of the device based on spiro-OMeTAD.

Sun and co-workers ${ }^{[31]}$ developed another two-arm SFXbased HTM termed with X59 (Figure 6) when demonstrating four-arm X60. The HOMO level, hole mobility and doped conductivity of X59 are quite close to those of spiro-OMeTAD. The PSC with the structure of $\mathrm{FTO} /$ compact $\mathrm{TiO}_{2} /$ meso-porous $\mathrm{TiO}_{2} /$ perovskite/X59/Au exhibited a PCE of up to $19.8 \%$, which is comparable to $20.8 \%$ of spiro-OMeTAD-based PSC under same conditions.

Taking full advantage of the multiple activation sites of SFX, Zhan and co-workers ${ }^{[32]}$ reported four SFX-based HTMs functionalized with electron-donating bis(4-methoxyphenyl)aniline or bis(4-methoxyphenyl)amine groups (Figure 6), named mp-SFX-3PA, mp-SFX-2PA, mm-SFX-3PA and mm-SFX-2PA (the same as $\mathrm{X60}$ ), respectively. The introduction of bis(4-methoxyphenyl)aniline leads to the deeper HOMO energy levels relative to the introduction of bis(4-methoxyphenyl)amine (Table 1). Whereas introducing the same substitutes at different active sites has a little effect on HOMO energy levels. The measurement of ultraviolet photoelectron spectroscopy (UPS) shows that the HOMO energy levels of mp-SFX-2PA and spiro-OMeTAD are -5.06 and $-4.98 \mathrm{eV}$, respectively. Compared with spiro-OMeTAD, mp-SFX-2PA can match much well with perovskite whose $\mathrm{HOMO}$ level is $-5.43 \mathrm{eV}$, which leads to a higher PCE (16.8\%) of PSC with the structure of $\mathrm{FTO} / \mathrm{TiO}_{2} /$ $\mathrm{MAPbl}_{3} / \mathrm{mp}-\mathrm{SFX}-2 \mathrm{PA} / \mathrm{Au}$ than that of PSC device based on spiro-OMeTAD (15.5\%).

Shen and co-workers ${ }^{[33]}$ synthesized a novel SFX-based organic small molecule with bis-triphenylamine groups, named BTPA-4 (Figure 6), and employed it as the hole selective layer 
Table 1 Physical and chemical properties of HTMs and PCEs of PSCs based on these HTMs

\begin{tabular}{|c|c|c|c|c|c|}
\hline Ref. & HTMs & $\mathrm{HOMO} / \mathrm{eV}$ & Conductivity $/\left(\mathrm{S} \cdot \mathrm{cm}^{-1}\right)$ & Mobility $/\left(\mathrm{cm}^{2} \cdot \mathrm{V}^{-1} \cdot \mathrm{s}^{-1}\right)$ & PCEs/\% \\
\hline \multirow[t]{3}{*}[6]{} & $\mathrm{X} 54$ & -5.30 & $3.26 \times 10^{-4}$ (doped state) & $8.25 \times 10^{-5}$ (pristine state) & 13.6 \\
\hline & $\times 55$ & -5.23 & $8.43 \times 10^{-4}$ (doped state) & $6.81 \times 10^{-4}$ (pristine state) & 20.8 \\
\hline & spiro-OMeTAD & -5.13 & $1.43 \times 10^{-4}$ (doped state) & $1.48 \times 10^{-4}$ (pristine state) & 18.8 \\
\hline \multirow[t]{4}{*}{ [27] } & $\mathrm{X} 22$ & -5.01 & $1.01 \times 10^{-4}$ (doped state) & $8.85 \times 10^{-5}$ (doped state) & 14.3 \\
\hline & $\mathrm{X} 26$ & -5.08 & $2.79 \times 10^{-4}$ (doped state) & $4.31 \times 10^{-4}$ (doped state) & 20.2 \\
\hline & $\times 36$ & -5.12 & $5.05 \times 10^{-4}$ (doped state) & $1.97 \times 10^{-4}$ (doped state) & 18.9 \\
\hline & spiro-OMeTAD & -5.07 & $1.08 \times 10^{-4}$ (doped state) & $1.26 \times 10^{-4}$ (doped state) & 18.3 \\
\hline \multirow[t]{2}{*}{ [28] } & X60 & - & $1.1 \times 10^{-4}$ (pristine state) & $1.9 \times 10^{-4}$ (pristine state) & 19.84 \\
\hline & spiro-OMeTAD & - & $1.5 \times 10^{-4}$ (pristine state) & $8.1 \times 10^{-5}$ (pristine state) & 20.8 \\
\hline \multirow[t]{5}{*}{ [30] } & SFX-MeOTAD & -5.16 & $1.4 \times 10^{-5}$ (pristine state) & - & 12.4 \\
\hline & SFX-TAD & -5.34 & - & - & - \\
\hline & SFX-TCz & -5.70 & - & - & - \\
\hline & SFX-TPTZ & -5.39 & - & - & - \\
\hline & spiro-OMeTAD & -5.14 & $1.6 \times 10^{-5}$ (pristine state) & - & 13.0 \\
\hline \multirow[t]{2}{*}{ [31] } & X59 & -5.15 & $1.9 \times 10^{-4}$ (doped state) & $5.5 \times 10^{-5}$ (pristine state) & 19.8 \\
\hline & spiro-OMeTAD & -5.13 & $1.5 \times 10^{-4}$ (doped state) & $8.1 \times 10^{-5}$ (pristine state) & 20.8 \\
\hline \multirow[t]{5}{*}{ [32] } & mp-SFX-3PA & -5.08 & - & $\begin{array}{l}4.0 \times 10^{-6} \text { (pristine state) } \\
3.3 \times 10^{-5} \text { (doped state) }\end{array}$ & 14.05 \\
\hline & mm-SFX-3PA & -5.08 & - & $\begin{array}{l}5.3 \times 10^{-6} \text { (pristine state) } \\
2.2 \times 10^{-5} \text { (doped state) }\end{array}$ & 13.25 \\
\hline & $m p-S F X-2 P A$ & -4.92 & - & $\begin{array}{c}3.0 \times 10^{-5} \text { (pristine state) } \\
\left.1.5 \times 10^{-4} \text { (doped state }\right)\end{array}$ & 16.77 \\
\hline & $m m-S F X-2 P A$ & -4.89 & - & $\begin{array}{l}8.7 \times 10^{-6} \text { (pristine state) } \\
9.9 \times 10^{-5} \text { (doped state) }\end{array}$ & 14.10 \\
\hline & spiro-OMeTAD & -4.83 & - & $\begin{array}{l}4.5 \times 10^{-6} \text { (pristine state) } \\
2.0 \times 10^{-5} \text { (doped state) }\end{array}$ & 15.45 \\
\hline [33] & BTPA-4 & -5.3 & - & - & 5.55 \\
\hline \multirow[t]{4}{*}{ [34] } & BTPA-4 & -5.35 & $\begin{array}{l}1.49 \times 10^{-4} \text { (pristine state) } \\
2.18 \times 10^{-4} \text { (doped state) }\end{array}$ & - & 9.21 (forward scan) \\
\hline & BTPA-5 & -5.37 & $\begin{array}{l}6.42 \times 10^{-5} \text { (pristine state) } \\
2.69 \times 10^{-4} \text { (doped state) }\end{array}$ & - & 11.30 (forward scan) \\
\hline & BTPA-6 & -5.34 & $\begin{array}{l}5.60 \times 10^{-5} \text { (Pristine state) } \\
2.47 \times 10^{-4} \text { (doped state) }\end{array}$ & - & $\begin{array}{l}11.57 \text { (forward scan) } \\
13.81 \text { (reverse scan) }\end{array}$ \\
\hline & spiro-OMeTAD & - & - & - & 13.25 (forward scan) \\
\hline \multirow[t]{5}{*}{ [35] } & XDB & -5.15 & - & $7.3 \times 10^{-5}$ (pristine state) & $\begin{array}{c}5.4 \text { (without } t \mathrm{BP} \text { ) } \\
15.5 \text { (with } t \mathrm{BP})\end{array}$ \\
\hline & XOP & -5.15 & - & $9.7 \times 10^{-5}$ (pristine state) & $\begin{array}{c}15.0 \text { (without } t \mathrm{BP} \text { ) } \\
15.9 \text { (with } t \mathrm{BP} \text { ) }\end{array}$ \\
\hline & XMP & -5.15 & - & $1.3 \times 10^{-4}$ (pristine state) & $\begin{array}{c}16.5 \text { (without } t \mathrm{BP} \text { ) } \\
16.9 \text { (with } t \mathrm{BP} \text { ) }\end{array}$ \\
\hline & XPP & -5.15 & - & $1.6 \times 10^{-4}$ (pristine state) & $\begin{array}{c}17.2 \text { (without } t \mathrm{BP} \text { ) } \\
17.7 \text { (with } t \mathrm{BP} \text { ) }\end{array}$ \\
\hline & spiro-OMeTAD & -5.13 & - & $8.2 \times 10^{-5}$ (pristine state) & $\begin{array}{l}5.5 \text { (without } t \mathrm{BP} \text { ) } \\
16.5 \text { (with } t \mathrm{BP} \text { ) }\end{array}$ \\
\hline \multirow[t]{5}{*}{ [36] } & SFX-TPAM & -5.21 & - & - & 10.23 \\
\hline & SFX-TPA & -5.57 & - & - & 3.83 \\
\hline & SFT-TPAM & -5.69 & - & - & 3.16 \\
\hline & SFT-TPA & -5.71 & - & - & 2.85 \\
\hline & Spiro-OMeTAD & -5.0 & - & - & 8.17 \\
\hline
\end{tabular}

(HSL) in colloidal quantum dots solar cells (CQDSCs) with the structure of $\mathrm{ZnO} / \mathrm{PbS}-\mathrm{QDs} / \mathrm{BTPA}-4 / \mathrm{Au}$. The perfect match of HOMO energy levels between BTPA-4 and PbS QDs leads to an unimpeded hole transport from PbS-QDs layer to Au electrode. Meanwhile, the higher LUMO energy level of the BTPA-4 can inhibit electron injection from PbS-QDs to $A u$ electrode effectively. Therefore, the PCE of CQDSCs with BTPA-4 was considerably improved relative to that of CQDSCs without BTPA-4. In addition, the PCE of CQDSCs based on BTPA-4 is also higher than that of CQDSCs based on spiro-OMeTAD under the same conditions. Subsequently, Shen et al. ${ }^{[34]}$ designed two triphenylamine-based HTMs with SFX skeleton, named BTPA-5 and BTPA- 6 (the same as mm-SFX-3PA, Figure 6 ) and employed them with BTPA-4 as HTMs in PSCs. The HOMO energy levels and conductivities doped with Li-TFSI and $t \mathrm{BP}$ are close to each other for BTPA-4, BTPA-5 and BTPA- 6 (Table 1). The PL spectra measurements show that BTPA-6 has a better hole extraction ability than BTPA-4 and BTPA- 5 . Therefore, the device based on BTPA- 6 exhibited a better solar cell performance (11.57\%) than those with BTPA-4 and BTPA-5. Meanwhile, the cell based on BTPA- 6 also exhibited a PCE of $14.4 \%$, which was nearly comparable to the cell based on spiro-OMeTAD (15.0\%). 


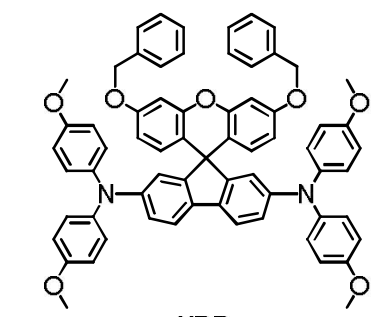

XDB

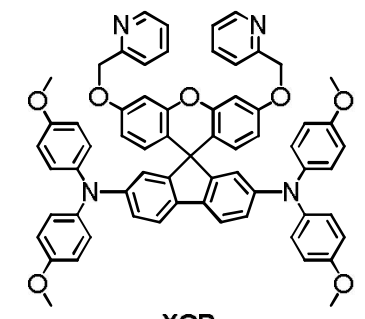

XOP<smiles></smiles>

XMP

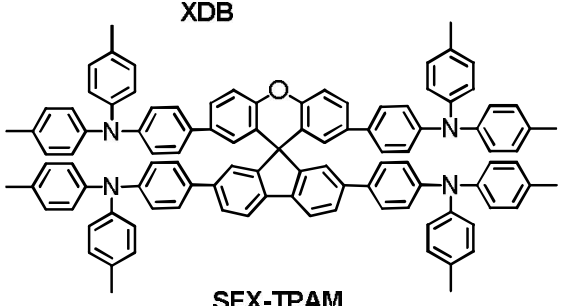

SFX-TPAM

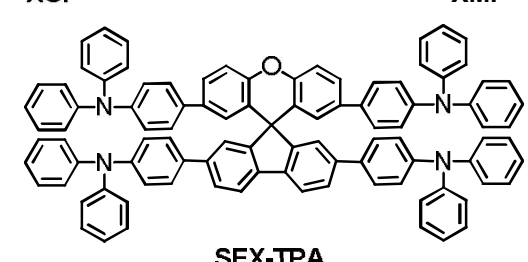

SFX-TPA

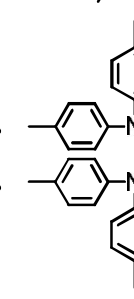<smiles></smiles>

XPP

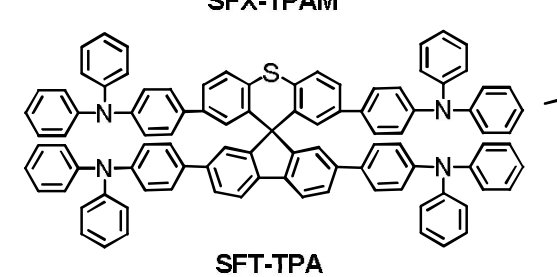

SFT-TPA<smiles>CCCCCCC(CCCCCC)(CCCCCC)c1cc(N(c2ccc(OC)cc2)c2ccc(OC)cc2)ccc1-c1ccc(N(c2ccc(OC)cc2)c2ccc(OC)cc2)cc1C1(CCCCCC)c2ccc(cc2)-c2ccc(N(c3ccc(OC)cc3)c3ccc(OC)cc3)cc21</smiles>

$\mathbf{X} 22$

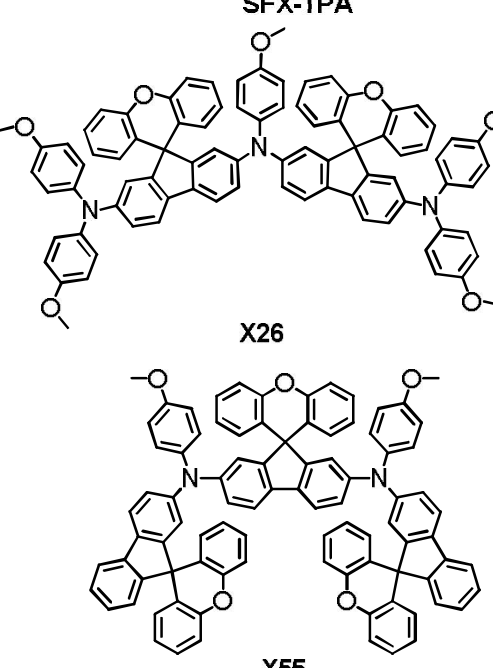

X55

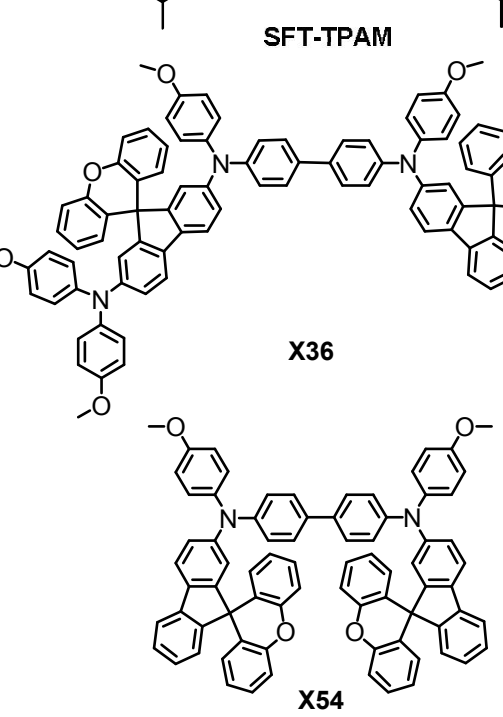

Figure 7 The structures of HTMs (2).

\section{Hole mobility and conductivity}

$\mathrm{Xu}$ et al. ${ }^{[35]}$ designed and synthesized a series of SFXbased HTMs replacing two bis(4-methoxyphenyl)amine arms with pyridine methoxyl or benzyloxy (XOP, XMP, XPP and $X D B$, Figure 7) on the basis of $X 60$. These compounds have the same HOMO energy levels of $-5.15 \mathrm{eV}$, which are almost the same as that of the Spiro-OMeTAD $(-5.13 \mathrm{eV})$ under the same conditions. However, the hole mobilities of XOP, XMP and XPP containing pyridine groups are higher than that of spiro-OMeTAD (Table 1). Steady-state PL and transient photocurrent decay measurements clearly indicate that pyridine-functionalized HTMs have comparably a much better hole extracting and transporting capability than spiro-OMeTAD. Therefore, these HTMs all exhibited higher PCEs of more than $14 \%$ (Table 1) in planar PSCs $\left(\mathrm{ITO} / \mathrm{SnO}_{2} / \mathrm{C}_{60} / \mathrm{CH}_{3} \mathrm{NH}_{3} \mathrm{Pbl} / 3 / \mathrm{HTM} / \mathrm{MoO}_{3} / \mathrm{Ag}\right)$ without 4-tert-butylpyridine (tBP) as additive than the PCE $(4.4 \%)$ of the device based on spiro-OMeTAD without $t B P$ under the same condition. Even in the PSCs with $t \mathrm{BP}$, these pyridine-functionalized HTMs also exhibited slightly superior performance than spiro-OMeTAD (Table 1).

In the reference [32], Zhan and co-workers also showed that both different substituent groups and different substitution positions have a different effect on the hole mobilities of HTMs. Among these HTMs, mp-SFX-2PA (Figure 6) has a higher hole mobility than mp-SFX-3PA, mm-SFX-3PA, mm-SFX-2PA and spiro-OMeTAD in both the pristine state and doped state (Table 1 ), which leads to an improved $J_{\mathrm{SC}}$ and FF so that the PSC based on mp-SFX-2PA exhibits a superior PCE relative to the PSCs based on other HTMs.

\section{Film-forming morphology}

Govindan et al. ${ }^{[36]}$ designed four HTMs based on SFX or spiro[fluorene-9,9'-thioxanthene] (SFT), termed SFX-TPAM, SFX-TPA, SFT-TPAM and SFT-TPA (Figure 7). Only SFXTPAM has a suitable HOMO energy level $(-5.21 \mathrm{eV})$ for $\mathrm{MAPbl}_{3}$ $(-5.4 \mathrm{eV})$ to transport its photo excited holes. The HOMO energy levels of the other three molecules are all deeper than that of $\mathrm{MAPb}_{3}$, which leads to even lower PCEs in PSCs than that of PSCs without hole-transporting layer (HTL). Though SFX-TPAM film has a lower hole mobility than that of spiro-OMeTAD film (Table 1), inverted PSC based on dopant-free SFX-TPAM HTL achieves a higher PCE of $10.23 \%$ in comparison to $8.17 \%$ of the PSC using dopant-free spiro-OMeTAD as a HTL. This is mainly due to the quality of the perovskite film-forming that the perovskite films deposited on more hydrophobic SFX-TPAM HTL has much larger grains compared to that coated on sipro-OMeTAD surface.

Sun and co-workers ${ }^{[27]}$ reported two new HTM molecules with SFX-based pendant groups, X26 and X36 (Figure 7), which present facile syntheses with high yields. Another molecule without SFX unit, X22 (Figure 7), was used as reference. The lower HOMO energy levels of X26 and X36 than that of X22 (Table 1) were favorable for higher open-circuit voltage $\left(V_{o c}\right)$ in PSC devices. Besides, X26 and X36 showed slightly higher hole mobility and conductivity than X22 without SFX pendant 
groups. However, it is the film-forming morphology of HTMs that determines the performance of PSCs. SEM monitoring results show that the X22- and X26- based films present a quite smooth surface and good coverage on top of the perovskite layer, but the $\mathrm{X} 36$-based film exhibits a porous structure with large voids. In addition, X26 forms a homogeneous capping layer, with efficient interfacial hole transfer, which is favorable to the $V_{\mathrm{oc}}$ and short-circuit current $\left(J_{\mathrm{sc}}\right)$ in the PSC devices. Therefore, the PSCs based on X26 exhibited a remarkable PCE of $20.2 \%$ in comparison to PCEs of $18.9 \%$ and $14.3 \%$ from X36- and X22-based devices, respectively.

Certainly, it is incomplete to evaluate the effect of HTMs on the performance of PSCs only from one aspect, and the comprehensive influence of several factors should be considered. However, it is difficult to develop a HTM molecule with suitable HOMO energy level, high hole mobility and conductivity as well as good film-forming ability at the same time. Sun and co-workers ${ }^{[6]}$ developed two SFX-based 3D oligomers, termed X54 and X55 (Figure 7), by further molecular engineering on the basis of their previous work $(X 59 \text { and } X 60)^{[28,31]}$ by introducing more than one SFX unit. Among the two HTMs, X55 shows a higher hole mobility and conductivity and better thermal stability than X54 and the well-known spiro-OMeTAD HTM. In addition, X55 has a much deeper HOMO energy level than spiro-OMeTAD. Meanwhile, X55, constructed with three bulky SFX units, shows a much better film-forming ability than Spiro-OMeTAD and X54 due to the excellent 3D structure, which offers higher solubility in the organic solvents used for spin coating. Therefore, PSC devices based on X55 exhibit an impressive PCE of $20.8 \%$, which is much higher than the PCEs of $18.8 \%$ and $13.6 \%$ for the spiro-OMeTAD and X54-based PSCs, respectively.

\section{Conclusion}

In summary, SFX building blocks have been becoming a new rising core in the field of HTMs by means of the low-cost, high-stability, multi-function and well-defined design principle as well as flexible tailoring, simultaneously. In most cases, using SFX as the core or introducing SFX unit to construct hole-transporting molecules can not only improve the solubility of HTMs in organic solvents and film-forming ability but also improve the hole mobility and conductivity of HTMs. However, it is more noteworthy that the diversity and low cost of building SFXbased HTMs make SFX-based HTMs more advantageous than other spiro materials. This is mainly due to the pioneering results of the simple one-pot synthesis of SFX. The excellent performance of SFX-based HTMs in PSCs is also fully demonstrated in this article, and the SFX-based HTMs are very potential for commercial application in high-efficiency PSCs. Due to the Shockley-Queisser limit, the theoretical efficiency of PSCs is about $31 \% .{ }^{[37,38]}$ Hence, the textured light-trapping structure and tandem PSCs device are advised. It is not only a challenge but also an opportunity for SFX-based HTMs to match these new-type device structures.

\section{Acknowledgement}

We express sincere gratitude to the Fundamental Research Funds for Central Universities (No. 2572018BC22).

\section{References}

[1] Swetha, T.; Singh, S. P. J. Mater. Chem. A 2015, 3, 18329.

[2] Kojima, A.; Teshima, K.; Shirai, Y.; Miyasaka, T. J. Am. Chem. Soc. 2009, 131, 6050.

[3] Kim, H. S.; Lee, C. R.; Im, J. H.; Lee, K. B.; Moehl, T.; Marchioro, A.; Moon, S. J.; Humphry-Baker, R.; Yum, J. H.; Moser, J. E.; Gratzel, M.; Park, N. G. Sci. Rep. 2012, 2, 591.
[4] Lee, M. M.; Teuscher, J.; Miyasaka, T.; Murakami, T. N.; Snaith, H. J. Science 2012, 338, 643.

[5] Burschka, J.; Pellet, N.; Moon, S. J.; Humphry-Baker, R.; Gao, P.; Nazeeruddin, M. K.; Gratzel, M. Nature. 2013, 499, 316.

[6] Xu, B.; Zhang, J. B.; Hua, Y.; Liu, P.; Wang, L. Q.; Ruan, C. Q.; Li, Y. Y.; Boschloo, G.; Johansson, E. M. J.; Kloo, L.; Hagfeldt, A.; Jen, A. K. Y.; Sun, L. C. Chem 2017, 2, 676.

[7] Song, Z. N.; Watthage, S. C.; Phillips, A. B.; Heben, M. J. J. Photon Energy 2016, 6, 022001.

[8] Lim, I.; Kim, E. K.; Patil, S. A.; Ahn, D. Y.; Lee, W.; Shrestha, N. K.; Lee, J. K.; Seok, W. K.; Cho, C. G.; Han, S. H. RSC Adv. 2015, 5, 55321.

[9] Xu, B.; Sheibani, E.; Liu, P.; Zhang, J. B.; Tian, H.; Vlachopoulos, N.; Boschloo, G.; Kloo, L.; Hagfeldt, A.; Sun, L. C. Adv. Mater. 2014, $26,6629$.

[10] Sung, S. D.; Kang, M. S.; Choi, I. T.; Kim, H. M.; Kim, H.; Hong, M.; Kim, H. K.; Lee, W. I. Chem. Commun. 2014, 50, 14161.

[11] Zhang, F.; Yi, C.; Wei, P.; Bi, X.; Luo, J.; Jacopin, G.; Wang, S.; Li, X.; Xiao, Y.; Zakeeruddin, S. M.; Grätzel, M. Adv. Energy Mater. 2016, 6, 1600401.

[12] Ameen, S.; Rub, M. A.; Kosa, S. A.; Alamry, K. A.; Akhtar, M. S.; Shin, H. S.; Seo, H. K.; Asiri, A. M.; Nazeeruddin, M. K. ChemSusChem 2016, 9, 10.

[13] Lv, S.; Han, L.; Xiao, J.; Zhu, L.; Shi, J.; Wei, H.; Xu, Y.; Dong, J.; Xu, X.; Li, D.; Wang, S.; Luo, Y.; Meng, Q.; Li, X. Chem. Commun. 2014, 50, 6931.

[14] Zheng, L.; Chung, Y. H.; Ma, Y.; Zhang, L.; Xiao, L.; Chen, Z.; Wang, S.; Qu, B.; Gong, Q. Chem. Commun. 2014, 50, 11196.

[15] Li, H.; Fu, K.; Hagfeldt, A.; Grätzel, M.; Mhaisalkar, S. G.; Grimsdale, A. C. Angew. Chem. Int. Ed. 2014, 53, 4085.

[16] Paek, S.; Zimmermann, I.; Gao, P.; Gratia, P.; Rakstys, K.; Grancini, G.; Nazeeruddin, M. K.; Rub, M. A.; Kosa, S. A.; Alamry, K. A.; Asiri, A. M. Chem. Sci. 2016, 7, 6068.

[17] Molina-Ontoria, A.; Zimmermann, I.; Garcia-Benito, I.; Gratia, P.; Roldan-Carmona, C.; Aghazada, S.; Grätzel, M.; Nazeeruddin, M. K.; Martín, N. Angew. Chem. Int. Ed. 2016, 55, 6270.

[18] Ganesan, P.; Fu, K.; Gao, P.; Raabe, I.; Schenk, K.; Scopelliti, R.; Luo, J.; Wong, L. H.; Grätzel, M.; Nazeeruddin, M. K. Energy Environ. Sci. 2015, 8, 1986.

[19] Abate, A.; Paek, S.; Giordano, F.; Correa-Baena, J.-P.; Saliba, M.; Gao, P.; Matsui, T.; Ko, J.; Zakeeruddin, S. M.; Dahmen, K. H.; Hagfeldt, A.; Grätzel, M.; Nazeeruddin, M. K. Energy Environ. Sci. 2015, 8, 2946.

[20] Jung, H. S.; Park, N. G. Small 2015, 11, 10.

[21] Bi, D.; Tress, W.; Ibrahim Dar, M.; Gao, P.; Luo, J.; Renevier, C.; Schenk, K.; Abate, A.; Giordano, F.; Correa Baena, J.; Decoppet, J.; Zakeeruddin, S. M.; Nazeeruddin, M. K.; Grätzel, M.; Hagfeldt, A. Sci. Adv. 2016, 2, e1501170.

[22] Ahn, N.; Son, D. Y.; Jang, I. H.; Kang, S. M.; Choi, M.; Park, N. G. J. Am. Chem. Soc. 2015, 137, 8696.

[23] Saliba, M.; Domanski, K.; Seo, J.; Ummadisingu, A.; Zakeeruddin, S. M.; Correa-Baena, J.; Tress, W. R.; Abate, A.; Hagfeldt, A.; Grätzel, M. Science 2016, 354, 206.

[24] Xie, L. H.; Liu, F.; Tang, C.; Hou, X. Y.; Hua, Y. R.; Fan, Q. L.; Huang, W. Org. Lett. 2006, 8, 2787.

[25] Clarkson, R. G.; Gomberg, M. J. Am. Chem. Soc. 1930, 52, 2881.

[26] Sun, M. L.; Xu, R. C.; Xie, L. H.; Wei, Y.; Huang, W. Chin. J. Chem. 2015, 33, 815.

[27] Zhang, J. B.; Xu, B.; Yang, L.; Ruan, C. Q.; Wang, L. Q.; Liu, P.; Zhang, W.; Vlachopoulos, N.; Kloo, L.; Boschloo, G.; Sun, L. C.; Hagfeldt, A.; Johansson, E. M. J. Adv. Energy Mater. 2018, 8, 1701209.

[28] Xu, B.; Bi, D. Q.; Hua, Y.; Liu, P.; Cheng, M.; Graetzel, M.; Kloo, L.; Hagfeldt, A.; Sun, L. C. Energy Environ. Sci. 2016, 9, 873.

[29] Xu, B.; Gabrielsson, E.; Safdari, M.; Cheng, M.; Hua, Y.; Tian, H.; Gardner, J. M.; Kloo, L.; Sun, L. C. Adv. Energy Mater. 2015, 10, 
1402340

[30] Maciejczyk, M.; Ivaturi, A.; Robertson, N. J. Mater. Chem. A 2016, 4, 4855.

[31] Bi, D. Q.; Xu, B.; Gao, P.; Sun, L. C.; Graetzel, M.; Hagfeldt, A. Nano Energy 2016, 23, 138.

[32] Liu, K.; Yao, Y.; Wang, J.; Zhu, L.; Sun, M. L.; Ren, B. Y.; Xie, L. H.; Luo, Y.; Meng, Q.; Zhan, X. W. Mater. Chem. Front. 2017, 1, 100.

[33] Zhang, Y.; Wu, G.; Mora-Sero, I.; Ding, C.; Liu, F.; Huang, Q.; Ogomi, Y.; Hayase, S.; Toyoda, T.; Wang, R.; Otsuki, J.; Shen, Q. J. Phys. Chem. Lett. 2017, 8, 2163.

[34] Wu, G.; Zhang, Y.; Kaneko, R.; Kojima, Y.; Sugawa, K.; Chowdhury, T. H.; Islam, A.; Shen, Q.; Akhtaruzzaman, M.; Noda, T.; Otsuki, J. Solar Rrl. 2017, 1, 1700096.
[35] Xu, B.; Zhu, Z.; Zhang, J.; Liu, H.; Chueh, C. C.; Li, X.; Jen, A. K. Y. Adv. Energy Mater. 2017, 7, 1700683.

[36] Govindan, V.; Yang, K. C.; Fu, Y. S.; Wu, C. G. New J. Chem. 2018 42, 7332.

[37] Sha, W.; Ren, X. G.; Chen, L. Z.; Choy, W. C. H. Appl. Phys Lett. 2015, 106, 221104

[38] Rajagopal, A.; Yang, Z. B.; Jo, S. B.; Braly, I. L.; Liang, P. W.; Hillhouse, H. W.; Jen, A. K. Y. Adv. Mater. 2017, 29, 1702140

Received August 13, 2019 Accepted September 21, 2019 